\title{
Home-based temperature measuring practices and their association with selected events of disease sequelae among children treated as dengue in a tertiary care setting in Sri Lanka
}

\author{
P.K.B. Mahesh ${ }^{1 *}$, S.M. Arnold ${ }^{2}$, M.W. Gunathunga ${ }^{3}$, A. Lathaharan ${ }^{4}$, A.M.N. Ariyarathne ${ }^{5}$, \\ N. Pannilahetti ${ }^{6}$, D.S.V. Mallawarachchi ${ }^{7}$
}

\begin{abstract}
Background

Correct and timely detection of fever, reduces the morbidity and mortality of dengue.

\section{Objective}

To describe the home-based temperature measuring practices and their association with selected events of disease progression among children treated as dengue in a tertiary care setting in Sri Lanka.

\section{Methods}

A descriptive cross sectional study was conducted in six general medical wards of the Lady Ridgeway Hospital for Children from July 2012 to December 2012 with a systematic sample of 425 children . Interviewer administered questionnaire and a data extraction sheet were used. Associations between fever detection practices and selected events of disease progression were analyzed (i.e. knowledge on fever, time of seeking treatment, need of intense monitoring, liver involvement, having an advanced grade for DHF and duration of stay)

\section{Results}

The availability of a thermometer at home is $47.5 \%(\mathrm{n}=201)$ with a majority having $152(75.6 \%)$ a mercury-in-built type. When detecting fever in the current episode, $136(32.2 \%)$ had used the thermometer. Having a thermometer $(p<0.01)$ and using a thermometer $(p<0.001)$ was associated with better knowledge on fever. Parents who used thermometers, had earlier treatment seeking $(\mathrm{p}=0.05)$ and lesser duration of stay $(\mathrm{p}<0.005)$.

\section{Conclusions}

Only one third of the parents used a thermometer to detect fever. Usage of a thermometer was associated with a better understanding of the disease process and had favorable outcomes in the current episode of dengue.

Key words: Dengue fever, temperature measurements, thermometer, treatment-seeking, outcome of disease

1. Senior Registrar in Community Medicine, Post Graduate Institute of Medicine, University of Colombo, Sri Lanka

2. Regional Director of Health Services, Office of Regional Director of Health Services, Colombo, Sri Lanka

3. Professor in Community Medicine, Faculty of Medicine, University of Colombo, Sri Lanka

4. Registrar in Community Medicine, Post Graduate Institute of Medicine, University of Colombo, Sri Lanka

5. Registrar in Community Medicine ,Post Graduate Institute of Medicine, University of Colombo, Sri Lanka

6. Consultant Community Physician, National Dengue Control Unit, Ministry of Health, Nutrition and Indigenous Medicine, Sri Lanka

7. Consultant Community Physician, Non Communicable Disease Unit, Ministry of Health, Nutrition and Indigenous Medicine, Sri Lanka

${ }^{*}$ Correspondence: buddhikamaheshpk@gmail.com

DOI: $10.4038 /$ jccpsl.v22i1.8084 


\section{Introduction}

Dengue is the most rapidly spreading mosquitoborne viral disease in the world (1). Children are at a higher risk of severe dengue. South East Asian and Western Pacific regions bear more than $75 \%$ of the global dengue burden. Dengue has become a major public health problem in Sri Lanka which is one of the four countries in the South East Asian Region in which dengue is a leading cause of hospitalization and death in children (1).

Unlike in other illnesses with symptoms of different systems in the body, in dengue, "fever" is the initial perception of ill-health and one of the main factors influencing its diagnosis. Hence the responses are mainly influenced by the stage the parents determine the child's fever. Timely interventions are utmost important in dengue management (2). As far as the parents are concerned, these practices include correct practices in detection of fever as well as correct practices in responding to fever.

Knowledge and practices of parents are found to be influencing the outcome in febrile illnesses. The World Health Organization has stated that "public education and community action" as important components in preventing dengue (3). National Plan of Action for Prevention and Control of Dengue Fever 2005-2009, the strategy number 02 of objective 01 was mentioned as "Development of health education messages for the community on early identification of dengue fever, early signs of DF/DHF and proper case management at household level."(4)

The methods of detecting fever among the general public is often improper (5). Correct fever detection practices include proper usage of a thermometer. In a research done in Turkey, it was found that of the parents, $56.5 \%$ stated that fever could be determined by touching the forehead (5). In a study done at Sri Jayawdenepura General Hospital in Sri Lanka, it was revealed that more than half of the parents had not measured fever with a thermometer (6).

Axilla has been the recommended site for temperature measurements in children under 5 years. Yet its value is less than that of oral temperature. Not concerning this variation of temperature of different sites would lead to incorrect determinations of febrile episodes (7).

The influence of knowledge and practices of fever on the outcome variables of dengue are not much studied in the local context in relation to dengue. Exploring these would lead to new insights on creating new effective interventions. These findings could be used for future interventional programmes to improve the knowledge and practices on determination of fever and health seeking behavior of parents which would ultimately have a positive impact on reducing the morbidity and mortality due to dengue/dengue hemorrhagic fever in Sri Lanka. Objective of this study was to describe the home-based temperature measuring practices and their association with selected events of disease sequelae among children treated as having dengue in the Lady Ridgeway Hospital for Children.

\section{Methods}

This was a descriptive cross sectional study with a follow up component. The study setting was six general pediatric medical wards of the Lady Ridgeway Hospital (LRH) for Children in which the children with dengue fever are routinely managed as in-ward patients. The study was carried out from July 2012 to January 2013. Study population was the caregivers (bystanders) of the in-ward children with dengue fever/dengue haemorragic fever. A suspected case of dengue by the ward staff, was verified 
by the investigators with the surveillance case definitions for notifiable diseases in Sri Lanka (8). Parents of the children at the Intensive Care Unit and those transferred from other hospitals were excluded.

Sample size was calculated using the formula (9); $n=Z 2 p(1-p) / d 2 . Z$ was taken as standard normal distribution value at $95 \%$ level, $\mathrm{d}$ (margin of error) was taken as $5 \%$ on either side and to get the maximum sample size $\mathrm{p}$ (estimation of proportion) was taken as $50 \%$ since local literature was not available for the study setting. With a response rate of $90 \%$, the required sample size was 425 at the data collection stage. Systematic sampling technique was used taking the admissions that met the eligibility criteria in each ward as the sampling frame. Data collection was carried out in all six medical wards in consecutive days until the required sample size was achieved.

A pre-tested interviewer-administered questionnaire and a data extraction form were used. Interviewer administered questionnaire included sections on socio-demographic data, knowledge on dengue fever, home-based temperature-measuring practices which were used in this episode and treatment seeking behaviour. The data-extraction sheet was used to obtain details about the selected four outcomes of the children treated as having dengue.

Data was entered into a SPSS version 17 datasheet and analyzed. Measures were taken to ensure the quality of data at designing, data collection and data analysis stages. The associations between categorical variables were tested using the chisquare test. When the outcome variable was numerical and a comparison was made between two groups, the independent sample $t$ test was used.

Ethical approval was obtained from the Ethics Review Committee of the Faculty of Medicine,
University of Colombo as well as from the Ethics Review Committee of the Lady Ridgeway hospital for Children.

\section{Results}

The study was carried out among 425 participants with a response rate is $99.5 \%$. A majority $(\mathrm{n}=270$, $63.8 \%$ ) of the admitted children were above 5 years of age. Another 80 (18.9\%) were between 3-5 years, $65(15.4 \%)$ were between 1-2 years and the rest $(\mathrm{n}=8,1.9 \%)$ were infants. It was found that over $97 \%(n=413)$ of the by standers were mothers while $10(2.4 \%)$ were fathers. The mean age (SD) of mothers was 35.6 (6.9) and their spouses were 38.8 (6.5). The mean age (SD) of the fathers who were staying with the child was 42.9 (6.6) and their spouses was 38.2 (8.4). Majority in the mothers' as well as the fathers' groups, had their education levels between grade 6 and General Certificate of Education ordinary level.

The availability of a thermometer at home was $47.5 \%(\mathrm{n}=201)$ out of which $152(75.6 \%)$ having a mercury-in-built thermometer and 49(24.4\%) digital thermometer. When detecting fever in the current episode, 136 (32.2\%) had used the thermometer (Table 1). This gives a probability that out of the families with a thermometer at home, only $67.7 \%$ had used it to detect of fever. Another 213 (50.4\%) have detected fever by touching the forehead alone.

Selected practices in relation to thermometer usage are described in Table 2. A majority $(n=126,92.6 \%)$ used axilla as the site to keep the thermometer. All who used the digital thermometer, kept it until a beep was heard. Out of the 112 who used the mercury-in-glass thermometer, $38(33.9 \%)$ kept it for more than 3 minutes whereas $25(22.3 \%) \mathrm{kept}$ it for less than two minutes. Others $(n=49,43.8 \%)$ kept it between 2-3 minutes. Of the respondents who detected fever by a thermometer $(n=136), 75 \%$ 
Table 1 Method used to detect the fever

\begin{tabular}{lcc}
\hline Method & Frequency & Percentage \\
\hline & 136 & 32.2 \\
1. Thermometer & 213 & 50.4 \\
2. Touching forehead & 8 & 1.9 \\
3. Touching the feet & 3 & 0.7 \\
4. Feeling warmth near the child & 63 & 14.9 \\
5. Combination of methods 2,3 and 4 & 423 & 100 \\
\hline \multicolumn{1}{c}{ Total } &
\end{tabular}

Table 1 Method used to detect the fever

\begin{tabular}{lcc}
\hline \multicolumn{1}{c}{ Practice } & Frequency & Percentage / \% \\
\hline Part of the body & 6 & 4.4 \\
Oral cavity & 126 & 92.6 \\
Axilla & 0 & 0 \\
Groin & 0 & 0 \\
Rectum & 136 & 100 \\
\hline \multicolumn{1}{c}{ Total } & 25 & 22.3 \\
\hline Duration of keeping thermometer* & 49 & 43.8 \\
$<2$ minute & 38 & 33.9 \\
$2-3$ minutes & 112 & 100 \\
$>3$ minutes & & \\
\hline \multicolumn{1}{c}{ Total } & 102 & 75.0 \\
\hline Using in between drug doses & 34 & 25.0 \\
\hline Yes & 136 & 100 \\
\hline No & & \\
\hline
\end{tabular}

*Excluded the 24, who used digital thermometer until a "beep noise" is heard 
$(\mathrm{n}=102)$ used it in between the drug doses. The balance $25 \%(n=34)$ did not use it for a second time after initial fever detection.

The knowledge of the parents on dengue fever was assessed with an interviewer administered questionnaire which was developed with expert inputs. It yielded a knowledge-score out of 50, per participant. With the decision of the panel of experts, the participants who had a score of $50 \%$ or above were categorized as with "satisfactory" knowledge. The mean knowledge score was 23.04 out of 50 which was expressed as $46.08 \%$. Table 03 summarizes the associations of knowledge-score with having a thermometer at home and having used it in the last episode.

The overall mean (SD) time period prior to treatment seeking was 1.67 (0.95) days. The perceived day of onset of fever was taken as day one. The mean (SD) number of days before treatment seeking from a health institution in private sector was $1.56(0.75)$ and from the government sector was 3.18 (1.45). Most patients sought care a day after onset of fever from private sector, and 3 days after onset from government sector. The mean (SD) day of getting a complete blood count was 3.51(1.27) days prior to admission and the mean duration before admission was 3.97 days from the onset. The respective mode for the day of getting the $\mathrm{CBC}$ and admission was 4 . The Table 3 summarizes the association between the average number of days from the time of onset for three selected aspects of treatment seeking. In the families that used a thermometer, the time duration before seeking treatment, for getting the first blood count and for getting admitted was shorter than the group that did not. The association is significant $(\mathrm{P}=0.05)$ for the day of treatment seeking.

Four selected outcomes were assessed; whether intense monitoring (admission to Intensive Care Unit or High Dependency Unit) was needed, whether liver enzymes (AST/ALT) levels were elevated, whether the duration of stay was more than 03 days and whether in DHF, grade was above II. Thirty percent $(n=127)$ of the admissions needed monitoring at HDU or ICU. Of the 328 in whom liver function tests (AST/

Table 3 Association between the level of knowledge versus having a thermometer and having used it in the last episode

\begin{tabular}{|c|c|c|c|c|c|c|c|}
\hline \multicolumn{8}{|c|}{ Knowledge } \\
\hline & \multicolumn{2}{|c|}{$\begin{array}{l}\text { Satisfactory } \\
\text { N (\%) }\end{array}$} & \multicolumn{2}{|c|}{$\begin{array}{c}\text { Not satisfactory } \\
\text { N (\%) }\end{array}$} & \multicolumn{2}{|c|}{$\begin{array}{l}\text { Total } \\
\text { N (\%) }\end{array}$} & \\
\hline $\begin{array}{c}\text { Having a } \\
\text { thermometer }\end{array}$ & & & & & & & $\begin{array}{l}\chi 2=34.61 \\
\text { df }-1\end{array}$ \\
\hline Yes & 130 & 64.7 & 71 & 35.3 & 201 & 100.0 & $\begin{array}{l}\mathrm{P}<0.01 \\
\mathrm{OR}=3.3\end{array}$ \\
\hline No & 80 & 36.0 & 142 & 64.0 & 222 & 100.0 & $\mathrm{CI}=2.2-4.8$ \\
\hline $\begin{array}{l}\text { Having used } \\
\text { the thermometer }\end{array}$ & & & & & & & $\begin{array}{l}\chi 2=37.68 \\
\text { df }-1\end{array}$ \\
\hline Yes & 97 & 71.3 & 39 & 28.7 & 136 & 100.0 & $\begin{array}{l}\mathrm{P}<0.001 \\
\mathrm{OR}=3.8\end{array}$ \\
\hline No & 113 & 39.4 & 174 & 60.6 & 287 & 100.0 & $\mathrm{CI}=2.5-6.0$ \\
\hline
\end{tabular}


Table 4 Association between usage of thermometer and average number of days elapsed from the time of onset for selected aspects of treatment seeking.

\begin{tabular}{|c|c|c|}
\hline & \multicolumn{2}{|c|}{ Used the thermometer } \\
\hline & Yes & No \\
\hline \multicolumn{3}{|c|}{ Day of seeking treatment } \\
\hline No & 117 & 258 \\
\hline Mean & 1.54 & 1.73 \\
\hline t value & \multicolumn{2}{|c|}{-1.96} \\
\hline Significance & \multicolumn{2}{|c|}{0.05} \\
\hline \multicolumn{3}{|c|}{ Day of getting the first blood count } \\
\hline No & 103 & 192 \\
\hline Mean & 3.49 & 3.52 \\
\hline $\mathrm{t}$ value & \multicolumn{2}{|c|}{-0.22} \\
\hline Significance & \multicolumn{2}{|c|}{0.82} \\
\hline \multicolumn{3}{|c|}{ Day of getting admitted } \\
\hline No & 123 & 244 \\
\hline Mean & 3.84 & 4.04 \\
\hline $\mathrm{t}$ value & \multicolumn{2}{|c|}{-1.43} \\
\hline Significance & \multicolumn{2}{|c|}{0.16} \\
\hline
\end{tabular}

ALT) were done, the values were elevated in $52.7 \% \quad(n=173)$. A duration of hospital stay of more than 03 days was needed for $67.5 \%$ $(\mathrm{n}=133)$. Of the 97 who were treated as DHF, $16.5 \%(n=16)$ were categorized as DHF III or IV.

The Table 4 summarizes the association between these four outcomes with "whether the thermometer was used in the current episode of fever". Having used a thermometer in the current episode of fever has a lesser risk $(\mathrm{OR}=0.5$, $95 \% \mathrm{CI}=0.3-0.8$ ) for prolonged hospital stay. The thermometer-used group had more favorable outcomes for liver involvement, need of intense monitoring and for the grade of DHF even though the associations were statistically not significant $(\mathrm{OR}<1 ; \mathrm{p}>0.05)$. 
Table 3 Association between selected factors and need of intense monitoring

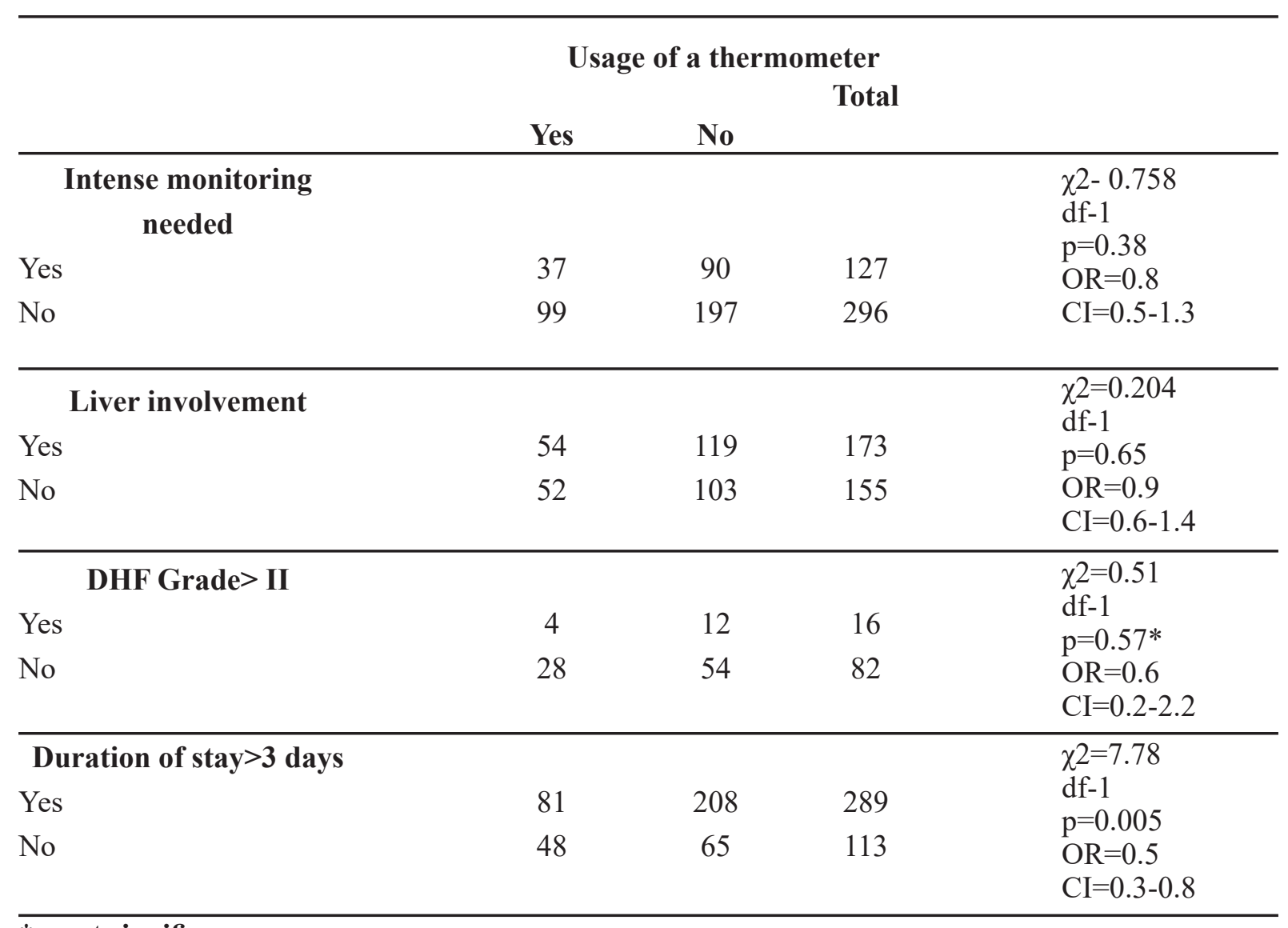

*exact significance

\section{Discussion}

Fifty three percent of the households had a thermometer at home. This is an improvement with the figure of $15 \%$ found in 2002 (Fernando, 2002). Of them, $67.7 \%$ had used it in detection of fever. In the study of Fernando, only $47 \%$ had used it indicating an increase in the availability as well as the usage of thermometer. This would have been attributed to the more emphasis done by the health care workers in usage of thermometers. Yet with better health inputs the proportion of people with a thermometer could be encouraged to use it.

As the Table 1 shows $50.4 \%$ had detected fever by touching the forehead whereas $32.2 \%$ used a thermometer. In Turkey in the study done by
Erek et al, 43.5\% used the thermometer. More emphasis must be given in future by interventions promoting the usage of thermometer by the general public. Among the families that use the thermometer, whether the correct technique is followed is a factor to be studied. Literature on the duration of insertion of thermometer to measure temperature is scarce and contradictory. In this study the proportion who kept it for more than 3 minutes was just $33.9 \%$. Globally there is a discouragement on the usage of mercuryin-glass thermometer. Hence the guidelines on these issues must be tailor made for the local setting as $75.6 \%$ of the thermometers used by the parents were mercury-in-built.

Cognition is said to be influencing the behavior. Therefore, knowledge should be reflected in 
practice. Statistically significant associations were obtained of the knowledge-score with presence of a thermometer and its usage $(p<0.01$ and $p<0.001$ respectively). Even though the causality of this association cannot be determined, better knowledge is seemingly associated with thermometer usage among the parents of children treated as dengue. Hence, improvement of knowledge on fever is likely improve the practices and vice versa.

In Sri Lankan, recent health messages emphasized the necessity of getting a blood counts done if fever persisted for more than 48 hours. The mean duration of getting a blood count done is 3.51 days. It would have been better if this period becomes lesser, partly because it takes some time until the report is available. This is more important in rural settings where the samples from private sector are taken in the morning to a regional center for testing by a "collector" who comes with the reports in the evening. The laboratory usually requests a contact telephone number in case results indicate a need for urgent intervention, but some parents either do not have a phone or those who do not understand what this urgency is, are reluctant to give it.

Usage of the thermometer was associated with lesser duration of stay in hospital probably indicating more accurate detection of fever and early admission reducing the morbidity. This view is further supported by those who measured temperature sought early treatment, getting the blood count done and getting admitted compared with those who do not use a thermometer. The difference between these two groups was significant $(\mathrm{p}=0.05)$ in relation treatment seeking.

Retrospective analysis of the gap between admission to hospital and the time of getting the blood count done, with the outcome measures show that mean time gaps are lesser for the group with favorable outcome. Though the association is not significant those who attend early seem to suffer fewer complications. This in turn is in agreement with the guidelines of the ministry emphasizing the value of timely intervention for reducing the morbidity and mortality. (Ministry of Health ,2010)

This study has few limitations. One was that not studying the mortality outcome. Information related to mortality remains mostly in the ICU. Due to logistic and ethics reasons patients in the ICU was excluded from the study. The protocols for sending the children to HDUs and ICUs are more or less similar in all 06 medical wards. But the bed availability inside the HDU and ICU becomes a factor in decision making. That factor was not considered in the study.

\section{Conclusions and Recommendations}

Only one third of the parents used a thermometer to detect fever. Having a thermometer at home had a significant positive association with the knowledge on fever. The knowledge on fever significantly influenced the usage of a thermometer. Usage of a thermometer was significantly associated with earlier treatment seeking from a health professional and with a lesser duration of hospital stay. It is recommended that more health education programs targeting general public on correct fever management be planned and implemented. More research should be encouraged in local setting relevant to fever management in dengue.

\section{Acknowledgements}

The authors would like to acknowledge the director and all the staff members of the general medical wards of the LRH for their support in carrying out this research.

\section{Conflicts of interest}

The authors had no conflicts of interest. 


\section{References}

1. World Health Organization (WHO) and Special Programme for Research and Training in Tropical Diseases (TDR). Dengue guidelines for diagnosis, treatment, prevention and control. Geneva: WHO and TDR, 2009.

2. Ministry of Health (MoH) and Sri Lanka College of Pediatricians (SLCP). 2010. National Guidelines: Guidelines on management of Dengue Fever and Dengue Hemarrhagic Fever in children and adolescents. Colombo: $\mathrm{MoH}$ and SLCP, 2010.

3. World Health Organization. Action against dengue; dengue day campaigns across Asia. http://www.wpro.who.int/ emerging_ diseases/documents/ Action Against Dengue.pdf.pdf. Geneva: WHO, 2011 (accessed on 03/01/2013)

4. Epidemiology Unit, 2005. National Plan of Action for Prevention and Control of Dengue Fever, 2005-2009, available at http://www.epid.gov.lk/web/images/ pdf/Circulars/latest draft-poa_for_dfdhf. pdf. Colombo: Epidemiology Unit, 2005 (accessed on 10/11/2012).
5. Erkek N, Senel S, Sahin M, Ozgur O, Karacan C. Parents' perspectives to childhood fever: comparison of culturally diverse populations. Journal of paediatrics and child health. 2010; 46 (10): p583-7.

6. Fernando SM, Wasala R. 2003 Study on the management of childhood fever at home. 116th anniversary academic sessions of programme and abstract book. Colombo: Sri Lanka Medical Association, 2003.

7. National Institute for Health and Clinical Excellence (NICE). Feverish illness in children: Assessment and initial management in children younger than 5 years: NICE, 2007.

8. Epidemiology Unit. Surveillance case definitions for notifiable diseases in Sri Lanka, 2nd edition. Colombo: Epidemiology Unit, 2011.

9. Lwanga SK, Lameshow S. Sample size determination in health studies; a practical manual. Geneva: World Health Organization, 1991. 